\title{
Landiolol hydrochloride ameliorates acute lung injury in a rat model of early sepsis through the suppression of elevated levels of pulmonary endothelin-1
}

Yujiro Matsuishi ${ }^{1}$, Subrina Jesmin ${ }^{1,2}$, Satoru Kawano ${ }^{1}$, Hideaki Sakuramoto ${ }^{1}$, Nobutake Shimojo ${ }^{1}$, Chishimba Nathan Mowa ${ }^{4}$, Shila Akhtar ${ }^{1}$, Sohel Zaedi ${ }^{1}$, Tanzila Khatun ${ }^{1}$, Yoshiya Tsunoda $^{3}$, Takumi Kiwamoto ${ }^{3}$, Nobuyuki Hizawa ${ }^{3}$, Yoshiaki Inoue ${ }^{1}$,Taro Mizutani ${ }^{1}$,

1. Department of Emergency and Critical Care Medicine, Faculty of Medicine, University of Tsukuba, Tsukuba, Ibaraki, Japan

2. Faculty of Health and Sports Science, Advanced Research Initiatives for Human High Performance (ARIHHP), University of Tsukuba, Tsukuba, Ibaraki, Japan

3. Department of Pulmonary Medicine, Faculty of Medicine, University of Tsukuba, Tsukuba, Ibaraki, Japan

4. Department of Biology, Appalachian State University, NC, USA

Running title: Landiolol hydrochloride's effect on pulmonary ET-1 levels in sepsis

Address requests for reprints to:

Dr. Satoru Kawano

Department of Emergency and Critical Care Medicine, Faculty of Medicine, University of Tsukuba, Tsukuba, Ibaraki, 305-8575, Japan.

Phone: +81-29-853-5633.

FAX: +81-29-853-3092

E-mail: kawano_s@md.tsukuba.ac.jp

Emails of authors:

Yujiro Matsuishi (0326yujiro@gmail.com)

SubrinaJesmin (jsubrina@gmail.com)

NobutakeShimojo (nokeshimojo@yahoo.co.jp)

Hideaki Sakuramoto (gongehead@yahoo.co.jp)

YoshiyaTsunoda (chabo448@yahoo.co.jp)

Takumi Kiwamoto (t-kiwamoto@md.tsukuba.ac.jp)

Nobuyuki Hizawa (nhizawa@md.tsukuba.ac.jp) 
Taro Mizutani(mizutani@md.tsukuba.ac.jp)

Satoru Kawano (kawano_s@md.tsukuba.ac.jp)

Yoshiaki Inoue (yinoue@md.tsukuba.ac.jp)

Chishimba N Mowa (mowacn@appstate.edu)

Abstract: Among the dysfunctions and pathologies associated with sepsis, the underlying molecular mechanisms of sepsis-induced acute lung injury (ALI) are poorly understood. Endothelin (ET)-1, a potent vasoconstrictor and pro-inflammatory peptide, is known to be involved in the pathogenesis of ALI in a rat model of sepsis. Here, we investigated whether landiolol hydrochloride, an ultra-short-acting $\beta$-blocker, plays a crucial role in ameliorating and attenuating LPS-induced ALI through modulation of the ET-1 system. Male Wistar rats at 8 weeks of age were administered with either saline or lipopolysaccharide (LPS) for three hours (3h) and some of the LPS-administered rats were continuously treated with landiolol for $3 \mathrm{~h}$. ALI was induced by LPS, including levels of both circulatory and pulmonary TNF- $\alpha$ and IL- 6 but [PaO2] was significantly decreased. LPS also induced a significant increase in levels of pulmonary ET-1 and ET-A receptor, but levels of ET-B receptor, which has vasodilating effects, were remarkably diminished. Further, LPS administration upregulated the pulmonary expression of HIF-1 $\alpha$. Finally, the treatment of LPS-administered rats with landiolol for $3 \mathrm{~h}$ ameliorated and prevented ALI, normalized the altered levels of pulmonary ET-1 and ET-A receptors. Landiolol also induced significant down-regulation of ET-B receptor in lung tissues in the early hours (phase) of sepsis. However, Landiolol treatment had no effect on the up-regulated inflammatory mediators (TNF- $\alpha$, IL-6) in both plasma and lung tissues during sepsis, and expression of pulmonary HIF-1 $\alpha$ also remained unchanged after landiolol treatment. Collectively, these data led us to conclude that landiolol may ameliorate sepsis-induced ALI via the pulmonary ET system.

\section{KEYWORD:}

Acute lung injury, Landiolol hydrochloride, Endothelin, Sepsis, Rat model 


\section{Introduction}

Sepsis is a critical life threatening condition with a definition that encompasses pathologic infection and physiological changes that are collectively known as systemic inflammatory response syndrome (SIRS) [1]. The mortality from sepsis and the associated complications is very high and is estimated to be about $30 \%$ [2], making it the second leading cause of death among patients admitted at non-coronary intensive care units [3]. To date, the pathogenesis of sepsis and its progression to multiple organ dysfunction syndrome (MODS) and the associated septic shock are poorly understood and thus have been the subject of investigations in the last several decades.

Acute lung injury (ALI) is a well-known and frequent complication of sepsis, and causes a significant number of sudden deaths (mortality) and morbidity [4-6]. For this reason, patients with ALI/ARDS account for a significant proportion of the intensive care unit (ICU) case load. ALI and acute respiratory distress syndrome (ARDS), the severe form of ALI, are characterized by an acute onset of severe hypoxia pulmonary infiltration [7], pulmonary hypertension, edema and deteriorated gas exchange [8]. Indeed, sepsis is a potential risk factor for ALI and ARDS [5].

To date, the molecular mechanisms underlying the pathogenesis of sepsis-induced ALI are poorly defined and understood. We do know that endothelin (ET)-1, a potent endogenous vasoconstrictor and pro-inflammatory peptide $[9,10]$, is actively involved in the pathogenesis of sepsis and the sepsis-associated organ dysfunction, as well as the associated complications [11], as evidenced by data from recent studies. Specifically, 
plasma levels of ET-1 are elevated in various sepsis animal models, including septic patients [12-15] and a clear correlation has been observed between ET plasma levels and morbidity/mortality in septic patients. These observations implicates ET in the pathogenesis of septic shock in human [16,17]. Further, ET-1 likely has a direct role in the development and subsequent severity of ALI by increasing the pressure of pulmonary microvasculature during the first phase or hours of sepsis $[18,19]$. Interestingly, endothelin blocker, namely tezosentan, ameliorates pulmonary hypertension, lung edema, cardiac dysfunction, and arterial hypoxemia in an ovine model of endotoxin-induced lung injury [20]. In contrast, intravenous infusion of ET-1 causes an elevation in pulmonary artery pressure and edema [21], implying a direct role of ET-1 in the pathogenesis of lung injury.

Landiolol hydrochloride, an ultra-short-acting and highly cardio-selective beta-1 blocker, with a half-life of $4 \mathrm{~min}$, has been used in treating several acute medical disorders, including arrhythmias, during heart surgery [22], acute myocardial infarction [23], acute decompensated heart failure [24], and refractory electrical storm [25]. Ultra-short-acting $\beta$-blockers, such as landiolol, can influence heart rate but exert minimal effect on cardiac function. Besides, the potential effects of landiolol on rhythm control, landiolol also plays a protective role against ALI in a rat model of lipopolysaccharide (LPS)-induced systemic inflammation, which is associated with a reduction in high mobility group box 1 (HMGB-1) [26]. Very recently, we have demonstrated that landiolol is effective in improving acute liver injury of sepsis through the modulation of TNF (tumor necrosis factor-alpha)- $\alpha$ [27]. In addition, we have also shown that ET-1 is highly upregulated in kidney and heart tissues in LPS-administered rats $[28,29]$ and landiolol has inhibitory effects on the upregulated levels of ET-1 in 
both of these tissues. However, it is not known whether landiolol will equally exert protective effects in lung tissues during sepsis through the alteration of the ET-1 system. Indeed, in our previous study we have already reported elevated ET-1 levels in lung tissues during sepsis [30].

In the present study, we investigated whether landiolol hydrochloride, an ultrashort-acting $\beta$-blocker, can play an important role in attenuating LPS-induced ALI through the modulation of pulmonary ET-1.

\section{MATERIALS AND METHODS}

Male Wistar rats (200-250 g, 8 weeks old) were used in all experiments in the current study. Sepsis was induced by the intra-peritoneal (IP) administration of bacterial LPS from Escherichia coli 055: B5 (15 mg/kg), dissolved in sterile saline, as described previously [31-33]. A dose $15 \mathrm{mg} / \mathrm{kg}$ of LPS has been shown to induce morphological injures in lung [34] as shown in our dose response study.

The total number of rats used in all the experiment was 45 , which was randomized into three groups, namely: Group 1 (control, $\mathrm{n}=15)$, group $2(\mathrm{LPS}, \mathrm{n}=15)$ and group 3 (LPS + landiolol hydrochloride, $\mathrm{n}=15)$. For group 1 , sterile saline $(2 \mathrm{ml} /$ body $)$ was administered at time $0 \mathrm{~h}$ and then the rats were killed after $3 \mathrm{~h}$ (control group). For group 2, LPS at a dose of $15 \mathrm{mg} / \mathrm{kg}$ was administered at time $0 \mathrm{~h}$, and then the rats were killed after 3h (sepsis group). In group 3, landiolol hydrochloride was administered intravenously $(100 \mu \mathrm{g} / \mathrm{kg} / \mathrm{min})$ for $15 \mathrm{~min}$ non-stop before LPS administration (landiolol treated sepsis group). The dose for landiolol was found to be the minimal 
dose for normalizing the LPS-induced hyperdynamic state in the acute (early) phase (hours) of sepsis, as reported in our past study [28].

Nembutal (sodium pentobarbital, IP, $80 \mathrm{mg} / \mathrm{kg}$ body weight, routinely used in our lab) was used to kill all the rats at $3 \mathrm{~h}$, at the end of the experimental protocol. Blood gas analysis was also performed in the current study. The blood samples were collected from a polypropylene tube catheter inserted into the left carotid artery for blood gas analysis, and then lung tissues were carefully harvested, snap-frozen in liquid nitrogen, and stored at $-80{ }^{\circ} \mathrm{C}$. All animals received proper care and the experimental procedures were approved by the Animal Care and Use Committee of University of Tsukuba prior to the study.

\section{Measurements of hemodynamic parameters}

Hemodynamic parameters were measured using a previously optimized methodology from our lab [27-35]. Briefly, rats were anesthetized with isoflurane inhalation $(1.5 \%, 11 / \mathrm{min})$ and a microtip pressure transducer catheter (SPC-320, Millar Instruments, Houston, TX, USA) was inserted into the left carotid artery at the end of the experimental protocol. Arterial blood pressure and heart rate (HR) were monitored with a pressure transducer (model SCK-590, Gould, Ohio, USA) and recorded with the use of a polygraph system (amplifier, AP-601G, Nihon Kohden, Tokyo, Japan; Tachometer, AT-601G, Nihon Kohden; and thermal-pen recorder, WT-687G, Nihon Kohden).

\section{Echocardiography}


Echocardiography was performed using a Vevo 2100 high-frequency ultrasound system (VisualSonics, Inc., Ontario, Canada), which includes an integrated rail system for consistent positioning of the ultrasound probe [36] at the end of the protocol. The fur from the chest was shaved with an electrical clipper and a gel. The animals were then connected to an electrocardiogram (ECG). An optimal parasternal long axis (LAX) cine loop (i.e. visualization of both the mitral and aortic valves, and maximum distance between the aortic valve and the cardiac apex) of $>1000$ frames/s was acquired using the ECG-gated kilohertz visualization technique. The probe was then rotated $90^{\circ}$ and positioned $6 \mathrm{~mm}$ below the mitral annulus. Three parasternal short-axis (SAX) M-mode sequences were stored. Percent fractional shortening (\% FS) was calculated in the M-mode image as $\mathrm{FS}=(\mathrm{EDD}-\mathrm{ESD}) / \mathrm{EDD}$, where $\mathrm{EDD}$ and $\mathrm{ESD}$ are the end-diastolic and end-systolic diameters, respectively.

\section{Lung wet-to-dry weight ratio}

Lung tissues were harvested, blotted dry and weighed in order to determine the weight of the lung in the wet state and the wet-to-dry weight ratio was calculated, as follows: the lung tissues were weighed; wrapped loosely in aluminum foil; dried in an oven at $80^{\circ} \mathrm{C}$ for $24 \mathrm{~h}$; and weighed again. Then the ratio lung wet-to-dry weight ratio was calculated.

\section{Histopathology examination}

After tissue harvest, the lungs were fixed in $4 \%$ buffered formalin solution, dehydrated, embedded in paraffin, and then sliced into 5-mm-thick sections to evaluate 
lung micro-morphological injury. After deparaffinization, tissue sections were stained using standard hematoxylin and eosin (HE) staining method.

\section{Enzyme-linked immunosorbent assay}

The concentration of each respective protein/peptide, as described below under the plasma/serum and pulmonary tissue extracts section, was determined using the following kits: serum and lung levels of tumor necrosis factor alpha (TNF- $\alpha$ ) and ET-1; plasma levels of IL-6 ( $\mathrm{R} \& \mathrm{D}$ Systems, Minneapolis, MN), according to the manufacturer's protocol. These kits are already validated for the measurement of rat samples in our lab.

\section{RNA preparation and real-time quantitative polymerase chain reaction}

Total RNA samples from lung tissues were isolated using the Acid guanidinium thiocyanatephenol chloroform extraction with RNeasy (Qiagen, Tokyo, Japan). After RNA isolation, quantification and DNase I treatment, RNA was reverse transcribed to cDNA using Omniscript RT and the first-strand cDNA synthesis kit (Qiagen). The reaction was performed at $37^{\circ} \mathrm{C}$ for $60 \mathrm{~min}$.

The mRNA expression levels of target genes were analyzed by real-time quantitative PCR using TaqMan probe and an ABI Prism 7700 sequence detector (PerkinElmer Applied Biosystems, Foster, CA). The gene-specific primers and TaqMan probes were synthesized from Primer Express version 1.5 software (PerkinElmer), according to the published cDNA sequences for each gene, as previously described $[32,37]$. The PCR mixture ( $25 \mu$ l total volume) consisted of forward and reverse primers 
for each gene (PerkinElmer) at $450 \mathrm{nM}$ each, FAM-labeled primer probes (PerkinElmer) at $200 \mathrm{nM}$, and TaqMan Universal PCR Master Mix (PerkinElmer). Each PCR amplification was performed in triplicate as follows: 1 cycle at $95{ }^{\circ} \mathrm{C}$ for $10 \mathrm{~min}$ and 40 cycles at $94{ }^{\circ} \mathrm{C}$ for $15 \mathrm{~s}$ and $60{ }^{\circ} \mathrm{C}$ for $1 \mathrm{~min}$. The quantitative values of target mRNAs were normalized by glyceraldehyde 3-phosphate dehydrogenase (GAPDH) mRNA, because GAPDH mRNA expressions were more stable among all the samples than other internal controls, such as $\beta$-actin and $18 \mathrm{~S}$ ribosomal RNA.

Primers and probes are as follows:

TNF- $\alpha$ forward: 5'-CCAGGAGAAAGTCAGCCTCCT-3', TNF- $\alpha$ reverse: 5'-TCATACCAGGGCTTGAGCTCA-3', and TNF- $\alpha$ probe: 5'-AGAGCCCTTGCCCTAAGGACACCCCT-3'; IL-6 forward primer: 5'-ACAGCCACTGCCTTCCCTAC-3', IL-6 reverse: 5'-TCTCATTTCCAAGATCTCCC-3', and IL-6 probe: 5'-CACAGAGGATACCACCCACA-3'; Hypoxia-inducible factor-1 alpha (HIF-1 $\alpha$ ) forward: 5'-CTATGGAGGCCAGAAGAGGGTAT-3', HIF-1 $\alpha$ reverse: 5'-CCCACATCAGGTGGCTCATAA-3', and HIF-1 $\alpha$ probe: 5'-AGATCCCTTGAAGCTAG-3', 
ET-1 forward: 5'-TCTACTTCTGCCACCTGGACAT-3';

ET-1 reverse: 5'-GAAGGGCTTCCTAGTCCATACG-3', and

ET-1 probe: 5'-CATCTGGGTCAACACTCC-3';

ET-A forward: 5'-GAATCTCTGCGCTCTCAGTGT-3',

ET-A reverse: 5'-GAGACAATTTCAATGGCGGTAATCA-3', and

ET-A probe: 5'-CAGGAAGCCACTGCTCT-3';

ET-B forward: 5'-GCTGGTGCCCTTCATACAGA-3',

ET-B reverse: 5'-CTTAGAGCACATAGACTCAACACTGT-3', and

ET-B probe: 5'-ATCCCCACAGAAGCCT-3';

GAPDH forward: 5'-GTGCCAAAAGGGTCATCATCTC-3',

GAPDH reverse: 5'-GGTTCACACCCATCACAAACATG-3', and

GAPDH probe: 5'-TTCCGCTGATGCCCC-3'.

\section{Statistical analysis}

The results were expressed as mean $\pm \mathrm{SE}$, and for parametric distribution, analysis

of variance test was used. For non-parametric distribution, Kruskal-Wallis test, followed 
by Steel-Dwass for multiple comparisons was used. A $P<0.05$ was considered statistically significant for all parameters.

\section{RESULT}

\section{Blood gas analysis results}

Table 1 shows the data of blood gas analysis in the current experimental setting.

$\mathrm{pH}$ was not significantly different among the three groups (Table 1). Arterial $\mathrm{PaO} 2$ was significantly decreased in LPS-administered rats compared to control group and landiolol treatment significantly increased the levels of arterial $\mathrm{PaO} 2$ in septic rats (Table 1). However, arterial $\mathrm{PaCO} 2$ was not significantly changed among the three groups. Blood lactate concentrations were elevated with LPS administration and $3 \mathrm{~h}$ treatment with landiolol partly normalized the elevated levels of lactate in LPS-administered rats (Table 1). $\mathrm{HCO}^{-}$and base excess were not significantly changed among the three groups (Table 1). 


\section{Hemodynamic changes in current experiments:}

We assessed the hemodynamic parameters in the rats after LPS administration. As shown in Table 2, both the levels of the systolic and diastolic blood pressure were significantly lower at $3 \mathrm{~h}$ after LPS administration compared to that of the control group and was unaffected by landiolol treatment. Landiolol significantly decreased the elevated heart rate in LPS-administered animals (Table 2).\% FS was also significantly increased (hyperkinetic) in LPS-administered group compared to that of control group, and a $3 \mathrm{~h}$ long treatment of LPS-administered rats with landiolol significantly reversed the elevated \% FS (Table 2).

\section{Morphological evaluation}

In the LPS-administered group, lung tissues showed thickening of alveolar, congestion formation and infiltration by neutrophils, but (these morphological abnormalities) were absent in the lung of the control group (Figure 1A). Landiolol treatment for $3 \mathrm{~h}$ significantly improved the lung congestion in septic rats (Figure 1A).

Wet-to-dry weight ratio of lung tissue was calculated in each study group in the present experiment. In the LPS-administered septic group, pulmonary wet-to-dry weight ratios 
were significantly higher compared to that of the control group and a $3 \mathrm{~h}$ treatment of septic animals with landiolol significantly normalized the pulmonary wet-to-dry ratio (Figure 1B).

\section{Evaluation of expression of ET-1 system in lung tissue}

Levels of ET-1 peptide were also evaluated in the current experimental setting (Figure 2A). ET-1 peptide expression was significantly increased in the lung tissue (about 2-fold) after LPS administration compared to the control group and landiolol treatment for $3 \mathrm{~h}$ significantly normalized the elevated levels of pulmonary ET-1 peptide in septic rats (Figure 2A). While levels of pulmonary ET-1 in septic rats was greatly ameliorated following the $3 \mathrm{~h}$ treatment by landiolol, landiolol treatment failed to normalize the elevated levels of plasma ET-1 in septic rats (Table 3). Prepro ET-1 mRNA levels were also elevated in lung tissue in the LPS-administered group compared to the control group. However, landiolol treatment normalized the elevated levels of prepro ET-1 mRNA in septic lung tissues (Figure 2B). Moreover, ET-A receptor mRNA expression in lung tissue was also elevated in the LPS administration group compared to the control group, and landiolol treatment normalized the elevated 
levels of ET-A receptor mRNA (Figure 2C) in septic rats. In contrast, ET-B receptor mRNA expression in lung tissue significantly decreased in the LPS administration group compared to the control group, and landiolol treatment failed to normalize the decreased levels of ET-B receptor mRNA in septic pulmonary tissues (Figure 2D).

\section{Evaluation of expression levels of inflammatory cytokine (TNF-alpha and IL-6)}

Both the upregulated plasma levels of potential inflammatory cytokines, TNF- $\alpha$ and IL-6 were unchanged with the treatment of landiolol in septic rats (Table 3). We also evaluated the expression patterns of mRNA and protein of TNF- $\alpha$, the key inflammatory cytokine in lung tissues during sepsis. Levels of both protein and mRNA (TNF- $\alpha$ ) were significantly higher in septic lungs compared to the control group and a 3 $\mathrm{h}$ treatment of septic rats with landiolol could not normalize the elevated levels of TNF in lungs (Figure 3A, 3B). In the current experimental setting, the expression levels of IL-6, another highly potent inflammatory cytokine, had parallel expression pattern as TNF- $\alpha$ (Figure 3C), which was upregulated in LPS-administered rats and was unchanged following landiolol treatment. 


\section{Evaluation of expression levels of HIF-1 $\alpha$}

In addition, to clarify whether LPS administration induces any hypoxic condition in pulmonary tissues, we examined the mRNA expression levels of HIF-1 $\alpha$ in lung tissue in present study. HIF-1 $\alpha$ mRNA expression levels were also increased in the LPS administration group in lung tissues, but landiolol treatment did not normalize the elevated pulmonary levels of HIF-1 $\alpha$ (Figure 3D).

\section{Discussion}

The present study is the first to demonstrate the normalization of elevated ET levels in rat pulmonary tissues by an ultrashort acting beta blocker (landiolol hydrochloride) during the early stages (hours) of sepsis. Further, this phenomenon (of landiolol on pulmonary ET-1 level) is also accompanied by an improvement in the morphology of the septic lung tissues. Here, we also show that pre-treatment of LPS-administered rats with landiolol for $3 \mathrm{~h}$ normalized both the diminished levels of $\mathrm{PaO} 2$ and enhanced levels of blood lactate in early sepsis. In contrast, these observed 
pulmonary ameliorations (molecular and morphological) in septic rats induced by landiolol are not associated with alteration in levels of inflammatory cytokines (TNF- $\alpha$, IL-6) and hypoxia marker (HIF-1) under the current experimental setting.

A previous study has reported that LPS increases heart rate and \% FS in rats $3 \mathrm{~h}$ post administration and that if these (septic) animals are treated with landiolol these altered conditions (HR and \% FS) are normalized [28,29]. It is interesting that while landiolol was able to decrease \% FS and HR in septic rats, as reported previously, it (landiolol) had no significant effect on systolic and diastolic blood pressure under the current experimental setting. In fact, landiolol's effect on blood pressure was lesser than esmolol, an ultra- short-acting $\beta$-blocker $[38,39]$. It is likely that landiolol's ability to attenuate the LPS-induced hyperdynamic state in septic rats may be linked to its (landiolol) partial normalization of elevated blood lactate concentrations in early sepsis.

The present study demonstrates landiolol's ability to improve $\mathrm{PaO} 2$ during the early phase (hours) of sepsis in rats, as well as reduction of congestion and normalization of the wet-to-dry ratio in lungs compared to the untreated group. These findings are significant since LPS has been shown to induce edema formation, 
infiltration of interstitial tissue by neutrophils, and reduction of alveolar spaces in lungs, $12 \mathrm{~h}$ post-treatment [40] and, importantly, these (same) morphological injuries diminished after administration of landiolol [26]. Thus, based on the current data, we speculate that landiolol is equally effective in eliminating acute septic-induced pulmonary tissue injuries through the reduction of congestion, and, ultimately, leads to a decrease in pulmonary wet-to-dry weight ratio and improvement in arterial $\mathrm{PaO}$.

ET-1 is a potent vasoconstrictor and pro-inflammatory peptide and has previously been implicated in the pathogenesis of sepsis and sepsis-induced MODS [41,42]. Therefore, the present data, showing an increase in the plasma levels of ET-1, are consistent with data obtained from earlier studies [35] from our lab and others $[16,17,43]$. This observed increase in levels of plasma ET-1 in septic animals may be due to a combination of enhanced ET-1 synthesis, as well as impairment in ET clearance in renal and pulmonary tissues [16]. ETs is also known to play an important role in the pathogenesis of sepsis-induced ALI and vascular failure [44] and for this reason ET blockers have been shown to have a protective effect in ALI [45-49] and MODS in sepsis. Because of these biological effects ETs have attracted significant 
research interest in the development of novel therapeutic and diagnostic tools for sepsis and other systemic inflammatory response syndromes (SIRS) and disorders $[12,50,51,52]$. In our present study, we also found the elevated ET-1 level in lung tissues at $3 \mathrm{~h}$ after LPS administration at hyperdynamic state in septic rats as consistent to past studies $[30,35]$. Lastly, although levels of pulmonary ET-1 were found to be elevated during the early phases (hours) of sepsis, its two receptors, ET-A and -B were differentially expressed, i.e., levels of ET-A receptor were up regulated while that of ET-B receptor were down regulated in lung tissue of septic rats. These differential expression patterns (of ET receptors) in septic lung were consistent with data from past studies $[30,35]$.

The present study is the first time to demonstrate the amelioration of an altered ET system and ET-A but not ET-B in lung tissue of septic rats following a $3 \mathrm{~h}$ treatment of landiolol. We intend to examine in depth the specific mechanisms underlying the differential effects of landiolol on the expression of pulmonary ET receptor subtypes in future using genetically altered/manipulated experimental animals, such as the ET receptor knockout mice. In wrapping up, we speculate that landiolol may normalize the 
altered ET-1 system using a similar pathway or mechanism as the non-selective ET receptor antagonist, bosentan [45], and it (landiolol) may also lead to the repair of lung injury in ALI in rat models, as demonstrated in the current study. However, future studies are required to provide more data that supports our current speculation.

Landiolol has been shown to be effective in preventing ALI in LPS administered rat through the attenuation of an inflammatory marker, HMGB-1 [26], as well in repairing lung injury in the early phases (hours) of sepsis. Surprisingly, landiolol could not normalize the up regulated levels of pulmonary TNF- $\alpha$ and IL-6, two potential pro-inflammatory cytokines, back to baseline levels in septic rats. Based on these current findings, it appears that the observed reversal effects of landiolol on the elevated levels of ET-1 in pulmonary tissue does not depend on the expression of TNF- $\alpha$ and IL-6 in septic rats. In contrast, following landiolol treatment, the liver tissue of septic rats gets repairs during the acute phase of liver injury and the up regulated levels of hepatic TNF- $\alpha$ are normalized in the same experimental setting as in the current study [27]. Thus it can be concluded that landiolol-induced repair in organ injury during the acute phase of sepsis involves the normalization of differential organ-specific signaling 
pathways. It is interesting to note that in both of our studies (current and past study [27]), landiolol failed to normalize the up regulated levels of plasma TNF- $\alpha$, while exerting differential effects on the levels of TNF- $\alpha$ in various organs of septic rats. Further, although elevated HIF-1 $\alpha$ has been shown to be normalized in kidney tissues of septic rats, in addition to the amelioration of the up regulated levels of renal ET-1 by landiolol treatment [28], the reversal effects of landiolol on the up regulated levels of pulmonary ET-1 and HIF-1 $\alpha$ had a different outcome in the present study from that of the renal tissues. The LPS-induced elevated levels of HIF-1 $\alpha$ in lung tissue demonstrated by the present study may imply the presence of hypoxia. Future studies are undoubtedly essential in dissecting the organ-specific effects of landiolol on various signaling pathways in septic rats, including the very early stages of sepsis. Of note, no significant change in blood pressure of LPS-administered rats was observed after landiolol treatment in the current study. These findings imply that the normalization of the elevated levels of pulmonary ET-1 in sepsis by landiolol is independent of blood pressure changes. Mechanistic studies in future are essential in exploring and uncovering the underlying mechanism of the current findings and in shedding more 
insights on the potential protective effects that landiolol may exert on the lung in early sepsis.

\section{Conclusion}

The present study demonstrates that landiolol hydrochloride, a selective ultra-short-acting $\beta$-blocker, ameliorates lung injury in a rat model during early sepsis. Landiolol possibly exerts such an effect through the suppression of elevated levels of pulmonary ET-1 but not levels of TNF- $\alpha$ and HIF-1 $\alpha$. These current findings may create news areas to explore beneficial clinical and preventive applications of landiolol in sepsis-induced multiple organ dysfunction syndrome, particularly in the acute phase of lung injury.

\section{Acknowledgements}

This work was supported in part by a Grant-in-Aid for Scientific Research B and C from the Ministry of Education, Culture, Sports, Science and Technology of Japan (22390334, 23592025, 23406037, 23406016, 23406029, 24406026, 25462812, 25305034 and 16k11394) and Japan Society for the Promotion of Science. 


\section{References}

1. Bone RC, Balk RA, Cerra FB, et al. Definitions for sepsis and organ failure and guidelines for the use of innovative therapies in sepsis. The ACCP/SCCM Consensus Conference Committee. American College of Chest Physicians/Society of Critical Care Medicine. Chest. 1992;101(6):1644-1655.

2. Angus DC, WT L-Z, Lidicker J, Clermont G, et al.Epidemiology of severe sepsis in the United States: analysis of incidence, outcome, and associated costs of care. Crit Care Med. 2001;29(7):1303-1310.

3. Parrillo JE, Parker MM, Natanson C, et al. Septic shock in humans. Advances in the understanding of pathogenesis, cardiovascular dysfunction, and therapy. Ann Intern Med. 1990;113(3):227-242.

4. Hudson LD, Milberg JA, Anardi D, et al.Clinical risks for development of the acute respiratory distress syndrome. Am J Respir Crit Care Med. 1995;151(2 Pt 1):293-301.

5. Rubenfeld GD, Caldwell E, Peabody E, et al. Incidence and outcomes of acute lung injury. N Engl J Med. 2005;353(16):1685-1693. 
6. Sevransky JE, Levy MM, Marini JJ. Mechanical ventilation in sepsis-induced acute lung injury/acute respiratory distress syndrome: an evidence-based review. Crit Care Med. 2004;32(11 Suppl):S548-S553.

7. Wheeler AP, Bernard GR. Acute lung injury and the acute respiratory distress syndrome: a clinical review. Lancet. 2007;369(9572):1553-1564.

8. Persson BPP, Boels PJ, Lövdahl C, et al. Endotoxin induces differentiated contractile responses in porcine pulmonary arteries and veins. J Vasc Res. 2011;48(3):206-218.

9. Mitaka C, Hirata Y, Nagura T, et al.Circulating endothelin-1 concentrations in acute respiratory failure. Chest. 1993;104(2):476-480.

10. Yanagisawa M, Kurihara H, Kimura S, et al. A novel potent vasoconstrictor peptide produced by vascular endothelial cells. Nature. 1988;332(6163):411-415.

11. Comellas AP, Briva A. Role of endothelin-1 in acute lung injury. Transl Res. 2009;153(6):263-271. 
12. Battistini B, Forget MA, Laight D. Potential roles for endothelins in systemic inflammatory response syndrome with a particular relationship to cytokines. Shock. 1996;5(3):167-183.

13. Kaszaki J, Wolfárd A, Boros M, Baranyi L, et al. Effects of antiendothelin treatment on the early hemodynamic changes in hyperdynamic endotoxemia. Acta Chir Hung. 1997;36(1-4):152-153.

14. Pan C, Wang J, Liu W, et al. Low tidal volume protects pulmonary vasomotor function from "second-hit" injury in acute lung injury rats. Respir Res. 2012;13(1):77.

15. Aleksandra P-P, Gorąca A. Influence of specific endothelin-1 receptor blockers on hemodynamic parameters and antioxidant status of plasma in LPS-induced endotoxemia. Pharmacol Rep. 2012;64(6):1434-1441.

16. Pittet JF, Morel DR, Hemsen A, et al. Elevated plasma endothelin-1 concentrations are associated with the severity of illness in patients with sepsis. Ann Surg. 1991;213(3):261-264. 
17. Weitzberg E, Lundberg JM, Rudehill A. Elevated plasma levels of endothelin in patients with sepsis syndrome. Circ Shock. 1991;33(4):222-227.

18. Albertini M, Clement MG, Hussain SNA. Role of endothelin ETA receptors in sepsis-induced mortality, vascular leakage, and tissue injury in rats. Eur $J$ Pharmacol. 2003;474(1):129-135.

19. Rossi P, Wanecek M, Konrad D, et al. Tezosentan counteracts endotoxin-induced pulmonary edema and improves gas exchange. Shock. 2004;21(6):543-548.

20. Kuklin VN, Kirov MY, Evgenov O V, et al. Novel endothelin receptor antagonist attenuates endotoxin-induced lung injury in sheep. Crit Care Med. 2004;32(3):766-773.

21. Filep JG, Sirois MG, Rousseau A,et al. Effects of endothelin-1 on vascular permeability in the conscious rat: interactions with platelet-activating factor. $\mathrm{Br} J$ Pharmacol. 1991;104(4):797-804.

22. Sakamoto A, Kitakaze M, Takamoto S, et al.Landiolol, an ultra-short-acting $\beta_{1}$-blocker, more effectively terminates atrial fibrillation than diltiazem after open 
heart surgery: prospective, multicenter, randomized, open-label study (JL-KNIGHT study). Circ J. 2012;76(5):1097-1101.

23. Hanada K, Higuma T, Nishizaki F, et al. Randomized study on the efficacy and safety of landiolol, an ultra-short-acting $\beta 1$-adrenergic blocker, in patients with acute myocardial infarction undergoing primary percutaneous coronary intervention. Circ J. 2012;76(2):439-445.

24. Kobayashi S, Susa T, Tanaka T, et al. Low-dose $\beta$-blocker in combination with milrinone safely improves cardiac function and eliminates pulsus alternans in patients with acute decompensated heart failure. Circ J. 2012;76(7):1646-1653.

25. Miwa Y, Ikeda T, Mera H, et al. Effects of landiolol, an ultra-short-acting beta1-selective blocker, on electrical storm refractory to class III antiarrhythmic drugs. Circ J. 2010;74(5):856-863.

26. Hagiwara S, Iwasaka H, Maeda H,et al. Landiolol, an ultrashort-acting beta1-adrenoceptor antagonist, has protective effects in an \{LPS-induced\} systemic inflammation model. Shock. 2009;31(5):515-520. 
27. Yoshino Y, Jesmin S, Islam M, et al. Landiolol Hydrochloride Ameliorates Liver Injury in a Rat Sepsis Model by Down Regulating Hepatic TNF-a. J Vasc Med Surg. 2015;03(02).

28. Ogura Y, Jesmin S, Yamaguchi N, et al. Potential amelioration of upregulated renal HIF-1alpha-endothelin-1 system by landiolol hydrochloride in a rat model of endotoxemia. Life Sci. 2014;118(2):347-356.

29. Seki Y, Jesmin S, Shimojo N, et al. Significant reversal of cardiac upregulated endothelin-1 system in a rat model of sepsis by landiolol hydrochloride. Life Sci. 2014;118(2):357-363.

30. Jesmin S, Yamaguchi N, Zaedi S, et al. Time-dependent expression of endothelin-1 in lungs and the effects of TNF- $\alpha$ blocking peptide on acute lung injury in an endotoxemic rat model. Biomed Res. 2011;32(1):9-17.

31. Jesmin S, Gando S, Matsuda N, et al. Temporal changes in pulmonary expression of key procoagulant molecules in rabbits with endotoxin-induced acute lung injury: elevated expression levels of protease-activated receptors. Thromb Haemost. 2004;92(5):966-979. 
32. Shimojo N, Jesmin S, Zaedi S, et al. Alterations of gene expressions of preproET-1 and ET receptors in brains of endotoxemic Sprague-Dawley rats. Exp Biol Med (Maywood). 2006;231(6):1058-1063.

33. Yamaguchi N, Jesmin S, Zaedi S, et al. Time-dependent expression of renal vaso-regulatory molecules in LPS-induced endotoxemia in rat. Peptides. 2006;27(9):2258-2270.

34. Jesmin S, Gando S, Zaedi S, et al. Differential expression, time course and distribution of four PARs in rats with endotoxin-induced acute lung injury. Inflammation. 2007;30(1-2):14-27.

35. Zaedi S, Jesmin S, Maeda S, et al. Alterations in gene expressions encoding preproET-1 and NOS in pulmonary tissue in endotoxemic rats. Exp Biol Med (Maywood). 2006;231(6):992-996.

36. Yang X, Sun C, Anderson T, et al. Assessment of spectral Doppler in preclinical ultrasound using a small-size rotating phantom. Ultrasound Med Biol. 2013;39(8):1491-1499. 
37. Maeda S, Miyauchi T, Iemitsu M, et al. Effects of exercise training on expression of endothelin-1 mRNA in the aorta of aged rats. Clin Sci. 2002;103 Suppl (s2002):118S - 123S

38. Ikeshita K, Nishikawa K, Toriyama S, et al. Landiolol has a less potent negative inotropic effect than esmolol in isolated rabbit hearts. $J$ Anesth. 2008;22(4):361-366

39. Sasao J, Tarver SD, Kindscher JD, et al. In rabbits, landiolol, a new ultra-short-acting beta-blocker, exerts a more potent negative chronotropic effect and less effect on blood pressure than esmolol. Can J Anaesth. 2001;48(10):985-989.

40. Li G, Zhou CL, Zhou QS, Zou HD. Galantamine protects against lipopolysaccharide-induced acute lung injury in rats. Braz J Med Biol Res. 2016;49(2):e5008.

41. Furian T, Aguiar C, Prado K, et al. Ventricular dysfunction and dilation in severe sepsis and septic shock: relation to endothelial function and mortality. J Crit Care. 2012;27(3):319.e9-e15. 
42. Piechota M, Banach M, Irzmanski R, et al. Plasma endothelin-1 levels in septic patients. J Intensive Care Med. 2007;22(4):232-239.

43. Takahashi K, Silva A, Cohen J, et al. Endothelin immunoreactivity in mice with gram-negative bacteraemia: relationship to tumour necrosis factor-alpha. Clin Sci. 1990;79(6):619-623.

44. Forni M, Mazzola S, Ribeiro LA, et al. Expression of endothelin-1 system in a pig model of endotoxic shock. Regul Pept. 2005;131(1-3):89-96.

45. Carpenter TC, Stenmark KR. Endothelin receptor blockade decreases lung water in young rats exposed to viral infection and hypoxia. Am J Physiol Lung Cell Mol Physiol. 2000;279(3):L547-L554.

46. Fujii Y, Magder S, Cernacek P, et al. Endothelin receptor blockade attenuates lipopolysaccharide-induced pulmonary nitric oxide production. Am J Respir Crit Care Med. 2000;161(3 Pt 1):982-989.

47. Patel S, Liu X, Liu M, et al. HJP272, a novel endothelin receptor antagonist, attenuates lipopolysaccharide-induced acute lung injury in hamsters. Lung. 2014;192(5):803-810. 
48. Trabold B, Pawlik M, Nietsch R, et al. Bosentan reduces oxidative burst in acid aspiration-induced lung injury in rats. Injury. 2009;40(9):946-949.

49. Zhang Z, Jian X, Zhang W, et al. Using bosentan to treat paraquat poisoning-induced acute lung injury in rats. PLoS ONE. 2013;8(10):e75943.

50. Kowalczyk A, Kleniewska P, Kolodziejczyk M, et al.The role of endothelin-1 and endothelin receptor antagonists in inflammatory response and sepsis. Arch Immunol Ther Exp (Warsz). 2015;63(1):41-52.

51. Paulus P, Jennewein C, Zacharowski K. Biomarkers of endothelial dysfunction: can they help us deciphering systemic inflammation and sepsis? Biomarkers. 2011;16 Suppl 1:S11-S21.

52. Wort SJ, Evans TW. The role of the endothelium in modulating vascular control in sepsis and related conditions. Br Med Bull. 1999;55(1):30-48. 
Table 1 Blood gas analysis parameters

\begin{tabular}{|c|c|c|c|c|c|c|}
\hline \multirow{2}{*}{$\begin{array}{l}\text { Parameters } \\
\mathrm{pH}\end{array}$} & \multicolumn{2}{|c|}{ Control } & \multicolumn{2}{|r|}{ LPS } & \multicolumn{2}{|c|}{ LPS+Landiolol } \\
\hline & 7.39 & \pm 0.01 & 7.37 & \pm 0.02 & 7.38 & \pm 0.01 \\
\hline $\mathrm{PaO} 2$ (torr) & 102.8 & \pm 2.2 & 84.2 & $\pm 4.7^{*}$ & 102.4 & $\pm 3.9^{\#}$ \\
\hline $\mathrm{PaCO} 2$ (torr) & 40.2 & \pm 1.4 & 40.9 & \pm 2 & 37.2 & \pm 2 \\
\hline Base Excess (mmol/l) & 0.5 & \pm 0.5 & -2.8 & $\pm-0.7^{*}$ & -3.9 & $\pm 0.7^{*}$ \\
\hline Lactate $(\mathrm{mmol} / \mathrm{l})$ & 1.4 & \pm 0.1 & 2.8 & $\pm 0.2^{*}$ & 2 & $\pm 0.1^{\#}$ \\
\hline $\mathrm{HCO}^{-}(\mathrm{mmMol} / \mathrm{l})$ & 25.3 & \pm 0.5 & 21.4 & $\pm 0.7^{*}$ & 20.7 & $\pm 0.8^{*}$ \\
\hline
\end{tabular}

Data are mean \pm SE: $* \mathrm{p}<0.05$ vs. Control; $\sharp \mathrm{p}<0.05$ vs. LPS

Abbreviations: LPS; lipopolysaccharide

Table 2 Hemodynamics and echocardiogram

\begin{tabular}{|c|c|c|c|c|}
\hline Parameters & Control & & LPS & LPS+Landiolol \\
\hline Systolic BP (mmHg) & $125.6 \pm 3.2$ & 93.2 & $\pm 6.6^{*}$ & $98.5 \pm 5^{*}$ \\
\hline Diastolic BP (mmHg) & $99.8 \pm 3.1$ & 72.3 & $\pm 5.8^{*}$ & $75.5 \pm 4.8^{*}$ \\
\hline Heart Rate (bpm) & $468.7 \pm 19$ & 501.2 & $\pm 16.2^{*}$ & $439.9 \pm 6.5^{\#}$ \\
\hline Fractional Shortening (\%) & $40.3 \pm 1.3$ & 45.1 & $\pm 1.2^{*}$ & $41.2 \pm 1^{\#}$ \\
\hline
\end{tabular}

Data are mean $\pm \mathrm{SE} ; * \mathrm{p}<0.05$ vs. Control; $\sharp \mathrm{p}<0.05$ vs. LPS

Abbreviations: BP; blood pressure; LPS; lipopolysaccharide

Table 3 Plasma Tumor Necrosis Factor (TNF)- $\alpha$, Endothelin-1 (ET-1) and Interleukin-6 (IL-6) levels

\begin{tabular}{lrrrr}
\hline & \multicolumn{1}{c}{ Control } & \multicolumn{2}{c}{ LPS } & \multicolumn{2}{c}{ LPS+Landiolol } \\
\hline TNF- (plasma, pg/ml) & $15.7 \pm 7$ & $180 \pm 40^{*}$ & $184.3 \pm 30.1^{*}$ \\
ET-1 (plasma, pg/ml) & $3.8 \pm 0.4$ & $34 \pm 3.3^{*}$ & $32.5 \pm 3.3^{*}$ \\
IL-6 (plasma, pg/ml) & $18.3 \pm 1.9$ & $29 \pm 3.3^{*}$ & $26.7 \pm 3.7^{*}$ \\
\hline
\end{tabular}

Data are mean $\pm \mathrm{SE} ; * \mathrm{p}<0.05$ vs. Control; $\# \mathrm{p}<0.05$ vs. LPS

Abbreviations: LPS; lipopolysaccharide 


\section{Figure legends}

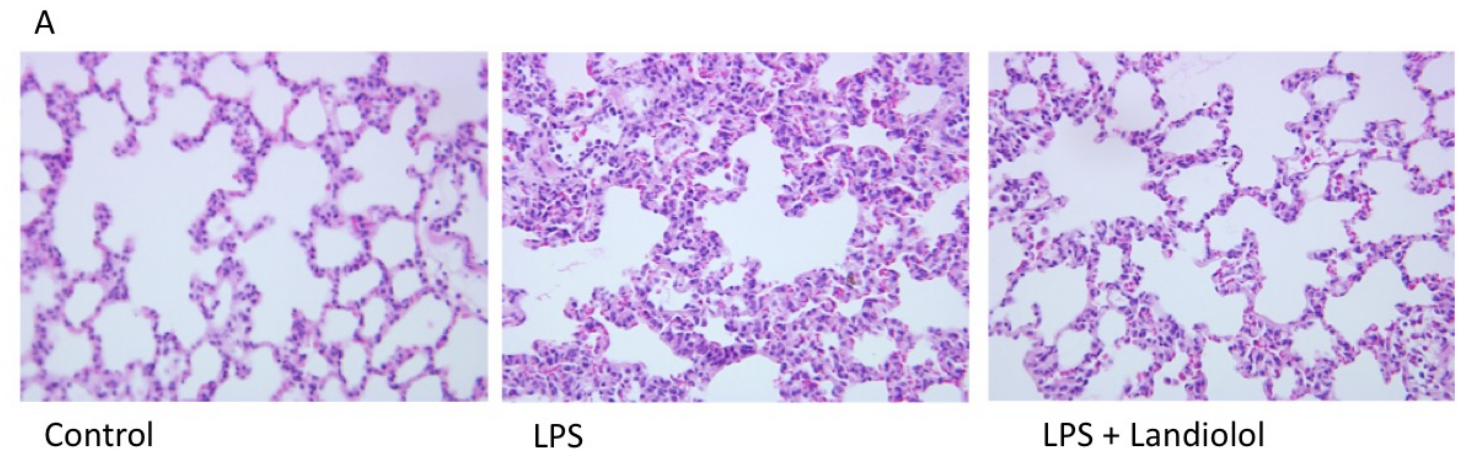

B

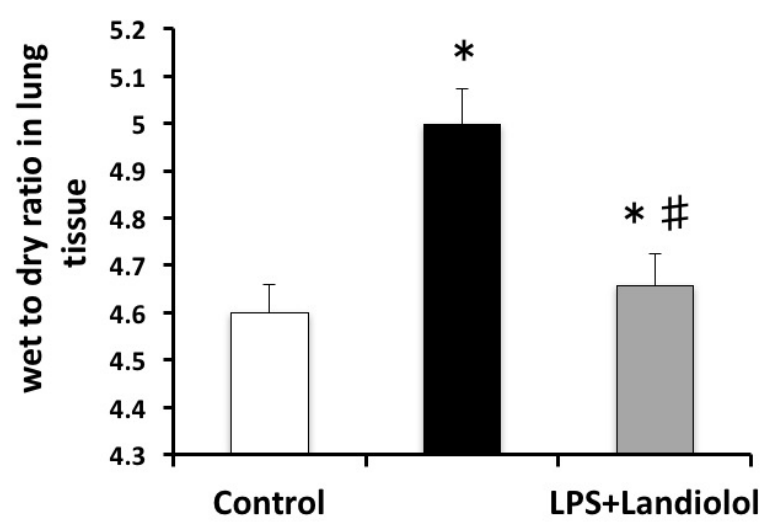

Figure 1: A; Morphological findings by hematoxylin and eosin staining (HE) inlung tissues in control rats, $3 \mathrm{~h}$ after lipopolysaccharide (LPS) administration, and $3 \mathrm{~h}$ after LPS plus landiolol hydrochloride administration. Magnification, $\times 320$.B; Pulmonary wet-to-dry weight ratio in control rats, $3 \mathrm{~h}$ after lipopolysaccharide (LPS) administration, and $3 \mathrm{~h}$ after LPS plus landiolol hydrochloride administration. Wet-to-dry weight ratios were determined for each experimental group. Values are mean $\pm \operatorname{SE}(n=5) . * p<0.05$ vs. Control, \# $\mathrm{p}<0.05$ vs. LPS 

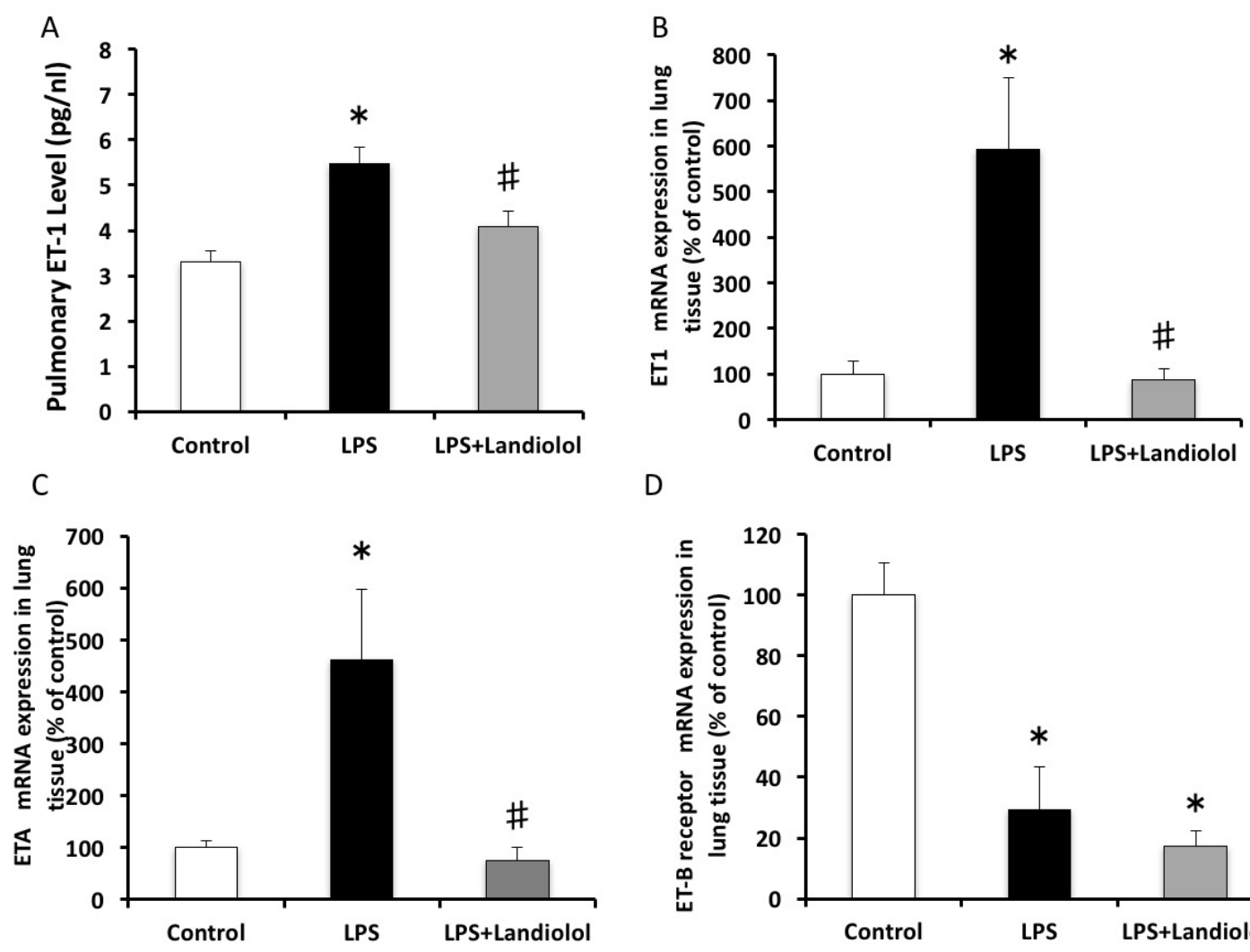

D

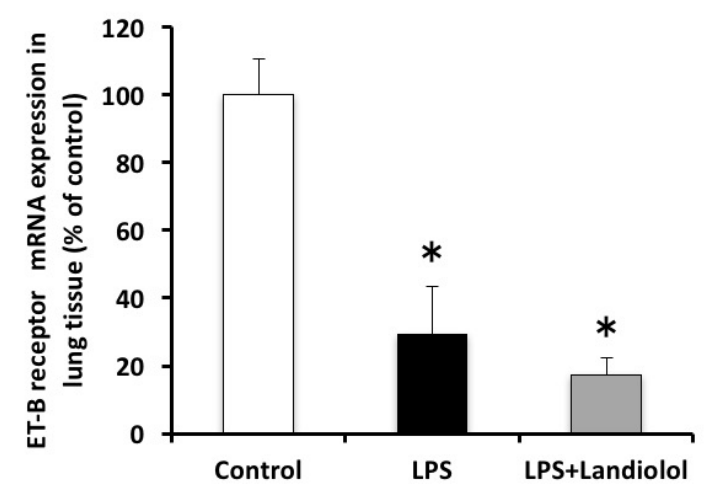

Figure 2: Peptide and mRNA expression levels of ET-1 (A and B),mRNA expression levels of ET-A (C), ET-B (D) receptors inlung tissues in control, 3h LPS-administered rats, and landiolol treated 3h LPS-administered rats.ET-1 peptide was measured by ELIZA. mRNA expression level was determined by Real Time PCR. The control was normalized as $100 \%$. Values are mean $\pm \mathrm{SE}(\mathrm{n}=15) .{ }^{*} \mathrm{p}<0.05$ vs. Control, $\# \mathrm{p}<0.05$ vs. LPS 

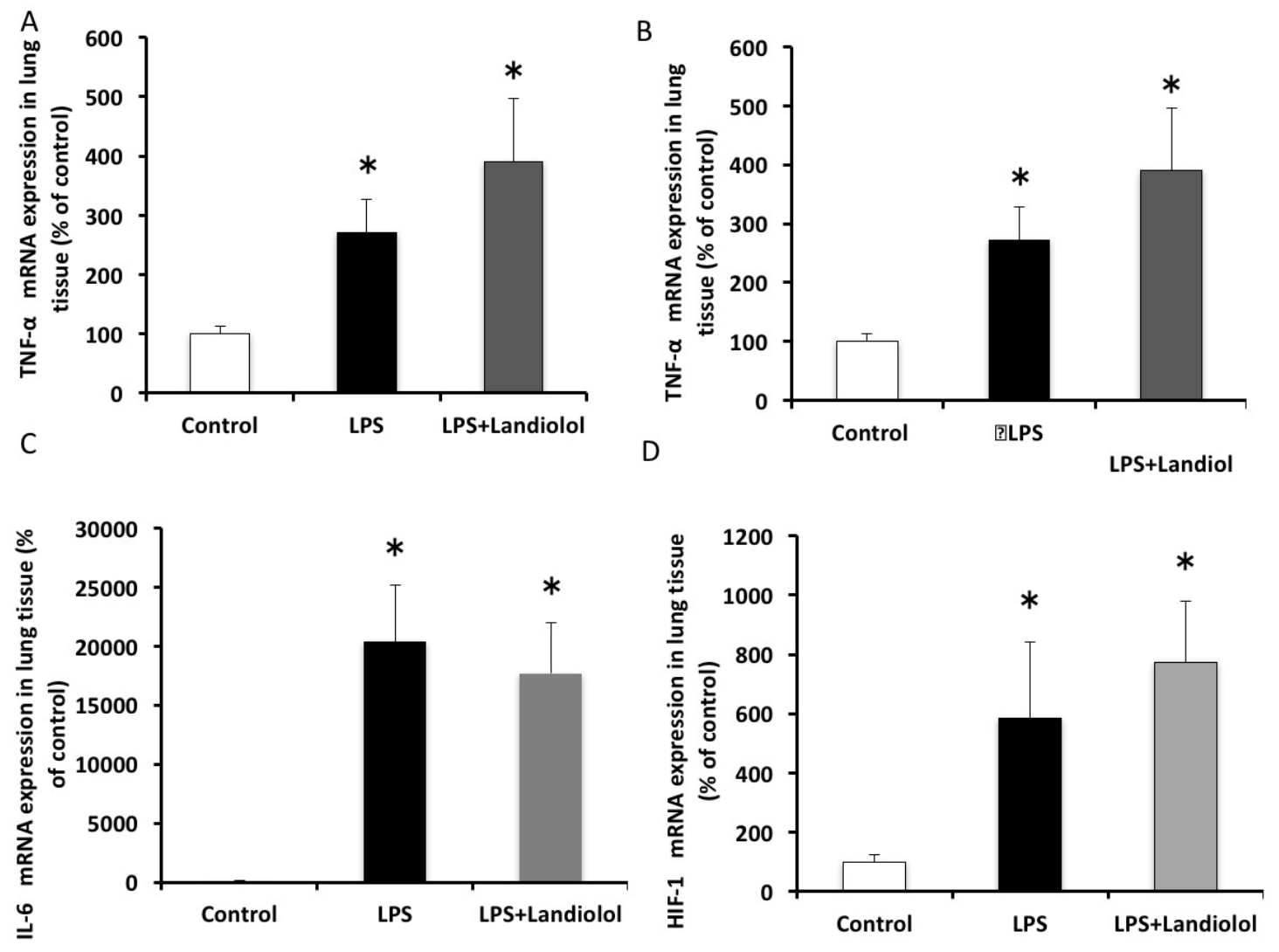

Figure 3: mRNA and protein expression levels of TNF- $\alpha$ (A and B), and mRNA expression levels of IL-6 (C), HIF-1 $\alpha$ (D) in lung tissues in control, 3h LPS-administered rats, and landiolol treated 3h LPS-administered rats. TNF- $\alpha$ protein was measured by ELIZA. mRNA expression level was determined by Real Time PCR. The control was normalized as $100 \%$. Values are mean $\pm \operatorname{SE}(n=15) .{ }^{*} \mathrm{p}<0.05$ vs. Control, \# $\mathrm{p}<0.05$ vs. LPS 